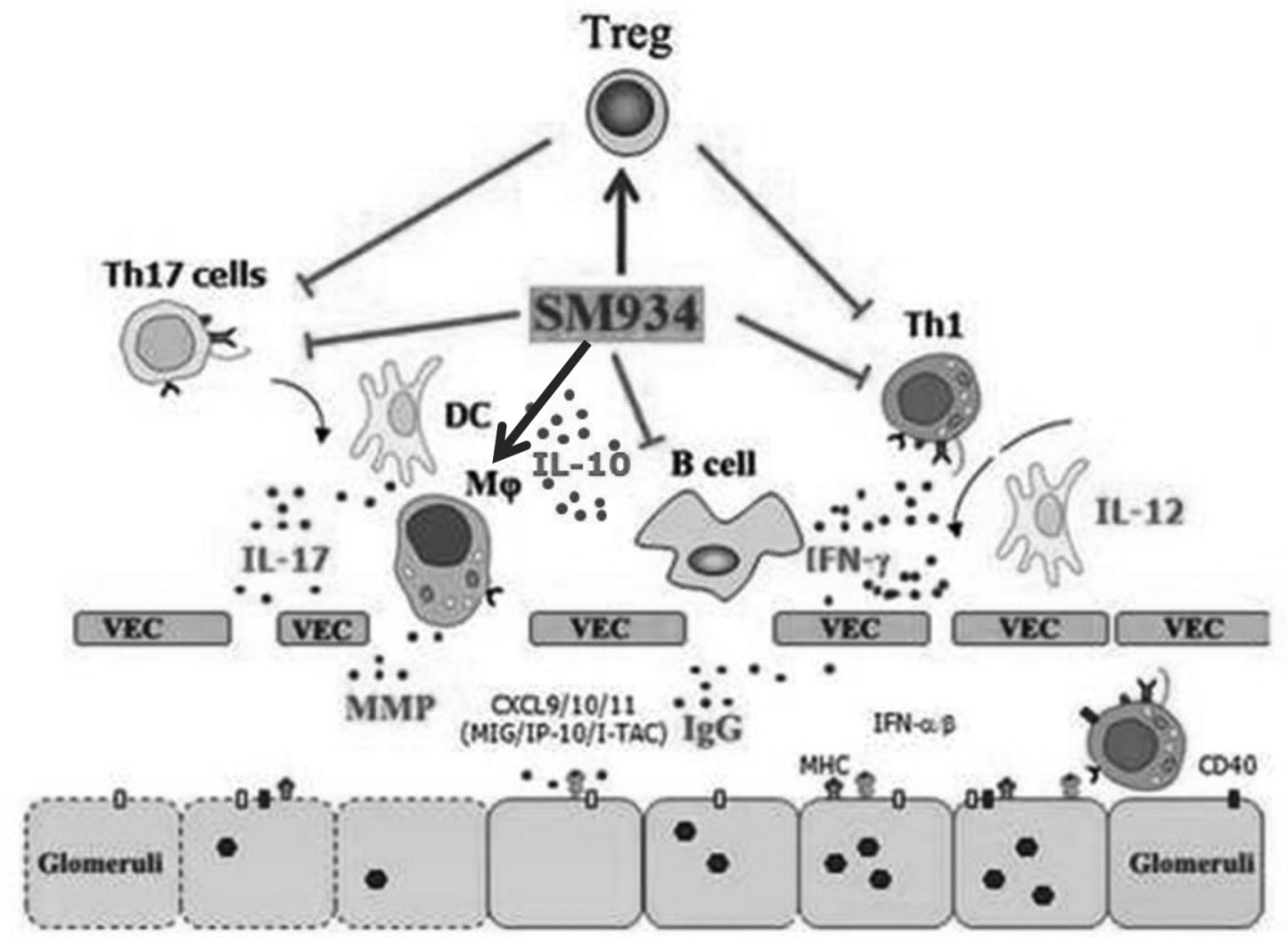

Abstract 68 Figure 1 SM934 restore immune homeostasis in SLE

Technology Major Project New Drug Creation and Manufacturing Program, China $(2014 Z \times$ 09101002) and National Key Basic Research Programme (973 Programme, 2014CB541906).

Artemisinin analogue SM934 treatment restore immune homeostasis in lupus-prone mice

\section{LUPUS IMPACT TRACKER CAN DIFFERENTIATE AMONG TREAT 2 TARGET OUTCOMES IN LUPUS PATIENTS WITH AND WITHOUT CONCURRENT FIBROMYALGIA}

${ }^{1}$ Meenakshi Jolly, ${ }^{2}$ Herve Devilliers, ${ }^{3}$ Iñigo Rua Figueroa, ${ }^{4}$ Javier Narváez-García, Jaime Calvo Alen, ${ }^{6}$ Maria Galindo Izquierdo, ${ }^{7}$ Francisco Javier López-Longo, ${ }^{8}$ Antonio Fernandez Nebro, ${ }^{9}$ Eva Tomero Muriel, ${ }^{10}$ Esther Uriarte Isacelaya, ${ }^{11}$ Mercedes Freire González*, ${ }^{12}$ Ricardo Blanco Alonso, ${ }^{13}$ Coral Mouriño Rodriguez, ${ }^{14}$ José C RosasGómez de Salazar, ${ }^{15} \mathrm{Gema}$ Bonilla-Hernán, ${ }^{16}$ Javier Narváez-García, ${ }^{17}$ Eva Salgado-Pérez, ${ }^{18}$ Lorena Expósito, ${ }^{1} J$ Joel Block, ${ }^{13}$ Jose Maria Pego Reigosa. ${ }^{1}$ Rush University Medical Center; ${ }^{2}$ University Hospital of Dijon; ${ }^{3}$ Hospital De Gran Canaria dr. Negrin; ${ }^{4}$ Hospital de Bellvitge; ${ }^{5}$ Hospital Universitario Araba; ${ }^{6}$ Hospital Universitario 12 De Octubre; ${ }^{7}$ Hospital General Universitario Gregorio Marañón; ${ }^{8}$ UGC de Reumatología, Instituto de Investigación Biomédica de Málaga (IBIMA) Hospital Regional Universitario de Málaga, Spain; ${ }^{9}$ Hospital De La Princesa; ${ }^{10}$ Hospital De Donostia; ${ }^{11}$ Hospital Juan Canalejo A Coruña (CHUAC); ${ }^{12}$ Hospital Marques de Valdecilla; ${ }^{13}$ Complexo hospitalario Universitario Vigo; ${ }^{14} \mathrm{Hospital}$ De Marina Baixa; ${ }^{15}$ Hospital De La Paz; ${ }^{16}$ Bellvitge University Hospital, Barcelona; ${ }^{17}$ Complejo Hospitalario De Ourense; ${ }^{18}$ Hospital Universitario de Canarias

\subsection{6/lupus-2019-|sm.69}

Background Remission and Low Disease activity state (LDAS) are the treat to target (T2T) outcomes used Systemic Lupus Erythematosus (SLE). Lupus Impact Tracker (LIT), a ten item unidimensional patient reported (PRO) tool with good psychometric properties and responsiveness including to composite response Index (SRI) and T2T outcomes. SLE patients often fibromyalgia (FM). We report discriminant validity of LIT to T2T outcomes in SLE patients stratified by FM from the largest European SLE registrycohort.

Methods Observational, multi-center data from 1364 adult patients with SLE meeting 1997 ACR criteria were obtained. This included demographics, LIT, disease activity-DA (SLEDAI) and medications. Remission off therapy (ROFT) was defined as $\mathrm{SLEDAI}=0$ without prednisone or Immunosuppressive/s. Remission on-therapy (RONT) was SLEDAI $=0$ and a prednisone dose $5 \mathrm{mg} /$ day and/or Immunosuppressive/s. LDAS (modified) was SLEDAI 4, prednisone dose $9 \mathrm{mg} /$ day and/or maintenance immunosuppressive/s. Non-optimal (NO) disease status was SLEDAI $>4$ and/or prednisone dose $>9 \mathrm{mg} /$ day and/or immunosuppressive/s in induction dose. LIT values were compared between T2T groups using mixed models among (a) all patients, (b) with and (c) without FM. For Model 1 : RONT and LDAS were combined (given relatively very low DA and lower prevalence of steroid use in LDAS) and compared with NO and ROFT. For model 2, all 4 T2T categories were used.

Results 1232/1364 (90\%) were women, and 95\% were Caucasian,76/1364 had FM. Median LIT (Q1, Q3) was 25 $(10,46.3)$. LIT scores were significantly different among T2T categories (table 1). Significant relevant differences were not evident between RONT and LDAS groups: 1) RONT had greater medication use than LDAS (prednisone $5-10 \mathrm{mg} /$ day use of $17.2 \%$ vs $8.3 \%$ )). 2) In LDAS, mean SLEDAI was 2, which can occur without clinical DA. RONT thus fared slightly worse than LDAS in patients evaluation of SLE impact. 
Abstract 69 Table 1 LIT values against T2T outcomes stratified by FM status in SLE

\begin{tabular}{|c|c|c|c|}
\hline Category & Estimated Mean LIT Score & standard error & $P$ value \\
\hline \multicolumn{4}{|c|}{ ALL $(n=1356)$} \\
\hline \multicolumn{4}{|l|}{ MODEL 1} \\
\hline Non Optimal & 34.57 & 0.98 & Ref \\
\hline LDAS/RONT & 28.22 & 0.65 & $<0.001$ \\
\hline ROFT & 24.61 & 1.1 & $<0.001$ \\
\hline \multicolumn{4}{|l|}{ MODEL 2} \\
\hline Non Optimal & 34.51 & 0.98 & Ref \\
\hline RONT & 30.43 & 0.94 & 0.0007 \\
\hline LDAS & 27.18 & 0.72 & $<0.001$ \\
\hline ROFT & 24.55 & 1.06 & $<0.001$ \\
\hline \multicolumn{4}{|c|}{ WITH FM ( $n=76)$} \\
\hline \multicolumn{4}{|l|}{ MODEL 1} \\
\hline Non Optimal & 56.76 & 4.5 & ref \\
\hline LDAS/RONT & 48.43 & 2.7 & $<0.001$ \\
\hline ROFT & 35.95 & 4.4 & $<0.001$ \\
\hline \multicolumn{4}{|l|}{ MODEL 2} \\
\hline Non Optimal & 56.64 & 4.51 & ref \\
\hline RONT & 53.95 & 5.8 & 0.64 \\
\hline LDAS & 45.77 & 5.06 & 0.03 \\
\hline ROFT & 35.83 & 5.84 & 0.006 \\
\hline \multicolumn{4}{|c|}{ WITHOUT FM $(n=1280)$} \\
\hline \multicolumn{4}{|l|}{ MODEL 1} \\
\hline Non Optimal & 33.4 & 1 & ref \\
\hline LDAS/RONT & 27.1 & 0.7 & $<0.001$ \\
\hline ROFT & 23.8 & 1.1 & $<0.001$ \\
\hline \multicolumn{4}{|l|}{ MODEL 2} \\
\hline Non Optimal & 33.3 & 0.99 & Ref \\
\hline RONT & 29.1 & 1.22 & 0.0006 \\
\hline LDAS & 26.13 & 1.04 & $<0.0001$ \\
\hline ROFT & 23.76 & 1.37 & $<0.0001$ \\
\hline
\end{tabular}

LIT was able to differentiate between T2T outcomes, and the scores moved in the anticipated direction. Though LIT scores for RONT were lower than NO in those with FM, they did not reach significance. This could potentially be secondary to small number of FM patients and use of 4 comparator categories.

Conclusions LIT can differentiate between T2T outcomes in SLE patients with and without FM. Given its extensive validation in various languages, regions, measurement equivalence, ease of use, acceptability by patients and physicians, and responsiveness to changes in assessments by physician (SLEDAI, PGA, SRI, T2T) and patient (HRQOL, SLAQ), the tool is indicated for routine patient care, research and clinical trials.

Funding Source(s): None

\section{R848 (RESIQUIMOD), A TLR 7/8 AGONIST, ACCELERATES DISEASE AND CAUSES A FATAL MYELOPROLIFERATIVE DISORDER IN NZM 2410 LUPUS- PRONE MICE}

${ }^{1}$ Jena Wirth, ${ }^{2}$ Sheryl Coutermarsh-Ott, ${ }^{1}$ Ivan Molano, ${ }^{3}$ Gary S Gilkeson, ${ }^{4}$ Melissa A Cunningham*. ${ }^{1}$ MUSC; ${ }^{2}$ Department of Biomedical Sciences and Pathobiology, VirginiaMaryland College of Veterinary Medicine; ${ }^{3}$ Division of Rheumatology and Immunology, Medical University of South Carolina; ${ }^{4}$ Medical University of South Carolina

\subsection{6/lupus-2019-Ism.70}

Background Spontaneous murine models of lupus-like disease are used to study the pathogenesis and genetic causes of SLE. Environmentally-induced lupus models (i.e. pristine-induced) are also valuable to study potential triggers of SLE. Both require extended observation. Some accelerants, (i.e. Type I IFN) are used to trigger earlier disease onset. We used a TLR $7 / 8$ agonist (R848), previously reported to induce lupus-like

Abstract 70 Figure 1 Treatment with Resiquimod (RQ) is associated with a marked proliferation of mononuclear cells consistent with histiocytic sarcoma. In acetone treated mice, the normal lymphoid follicular architecture of the spleen is maintained (top, low mag). In these animals, there are rare, scattered cells within the lymphoid follicles that exhibit strong staining for F4/80, consistent with histiocytic cells (bottom). In animals treated with RQ, there is effacement of the normal lymphoid follicles by a proliferation of mononuclear cells. In these mice, $80-90 \%$ of spleen cells exhibit strong punctate to diffuse cytoplasmic staining with F4/80 (bottom) consistent with histiocytic cells. The top images are stained with H\&E. The bottom images are stained with an F4/80 antibody using a red chromogen and DAB counterstain. Treatment of lupus prone mice with R848/ resiquimod (RQ) is associated with a marked proliferation of mononuclear cells in spleen consistent with histiocytic sarcoma. 